\title{
Minding The Gap: Severe Anion Gap Metabolic Acidosis Associated With 5-Oxoproline Secondary To Chronic Acetaminophen Use
}

Claudia Frankfurter, Kevin Venus, and David W. Frost

\section{About the Authors}

Claudia Frankfurter and Kevin Venus are with the Department of Medicine, University of Toronto, Toronto, ON, Canada. David W. Frost is with the Division of General Internal Medicine, Toronto Western Hospital, Toronto, ON, Canada. Correspondence can be directed to David Frost: david.frost@uhn.ca.

Submitted: January 9, 2019. Accepted: January 18, 2019. Published: November X, 2019. DOI: 10.22374/cjgim.v14i4.339

\begin{abstract}
An 89-year-old man with multiple comorbidities presented to the emergency department with diffuse abdominal pain and dyspnea. He was found to have a severe anion-gap metabolic acidosis with the normal osmolar gap. An initial panel of investigations for common causes of anion-gap metabolic acidosis was unremarkable. Further history revealed long-term daily acetaminophen use. A presumptive diagnosis of 5-oxoprolinemia secondary to chronic acetaminophen use was made. Despite supportive care, the patient did not survive. There is emerging literature on elevated anion gap metabolic acidosis induced by the accumulation of 5-oxoproline, an intermediate organic acid in the gamma-glutamyl cycle. A quantitative profile of urinary organic acids to measure 5-oxoproline is valuable in confirming the diagnosis. Treatment is largely supportive, consisting of cessation of acetaminophen, alkali therapy, and $\mathrm{N}$-acetylcysteine. Clinicians should consider 5-oxoprolemia in patients who present with an otherwise unexplained anion gap metabolic acidosis and a history of chronic acetaminophen use.
\end{abstract}

\section{RESUME}

Un homme de 89 ans souffrant de comorbidités multiples s'est présenté à l'urgence avec douleur abdominale diffuse et dyspnée. On a découvert qu'il souffrait d'une acidose métabolique grave à anions nuls avec un écart osmolaire normal. Un premier groupe détudes sur les causes courantes d'acidose métabolique à intervalle anionique n’a pas été remarquable. D'autres antécédents ont révélé une utilisation quotidienne à long terme de l'acétaminophène. Un diagnostic présumé de 5-oxoprolinémie secondaire à l'utilisation chronique d’acétaminophène a été posé. Malgré des soins de soutien, le patient n’a pas survécu. Il existe une littérature émergente sur l'acidose métabolique à intervalle anionique élevé induite par l'accumulation de 5-oxoproline, un acide organique intermédiaire dans le cycle gamma-glutamyle. Un profil quantitatif d'acides organiques urinaires pour mesurer la 5-oxoproline est utile pour confirmer le diagnostic. Le traitement est largement favorable, consistant en l'arrêt de l'acétaminophène, un traitement alcalin et de la $\mathrm{N}$-acétylcystéine. Les cliniciens devraient envisager l'administration de 5-oxoprolemia chez les patients qui présentent une acidose métabolique par gap anionique autrement inexpliquée et des antécédents d'utilisation chronique d'acétaminophène. 


\section{Case}

An 89-year-old man with multiple medical comorbidities was admitted through the emergency department for a 1-day history of diffuse abdominal pain, nausea, and non-bloody emesis. He also reported constipation and was unable to tolerate oral intake. He had developed resting dyspnea in the preceding week and noted marked weakness. A review of systems was otherwise non-contributory. In light of the anion gap metabolic acidosis, a detailed history of ingestions was taken, and there was no history of toxic alcohol use in any form.

His medical history was significant for heart failure with preserved ejection fraction, atrial fibrillation, tricuspid regurgitation, chronic obstructive pulmonary disease requiring supplemental home oxygen, pulmonary hypertension, obstructive sleep apnea, stage IA lung cancer treated with radiation, bladder cancer treated with endoscopic resection, monoclonal gammopathy of undetermined significance, stage III chronic kidney disease, cirrhosis secondary to non-alcoholic steatohepatitis, esophagitis with prior upper gastrointestinal hemorrhage, choledocholithasis, multiple small bowel obstructions, inguinal hernia, gout, and heparin-induced thrombocytopenia. He was an ex-smoker with a 50 pack-year smoking history. He did not consume ethanol, nor did he use illicit drugs or herbal supplements. He was taking acetaminophen for osteoarthritis-related pain.

His prescribed medications were apixaban, furosemide, rosuvastatin, indacaterol-glycopyrronium, salbutamol, ipratropium bromide, ferrous fumarate, pantoprazole, allopurinol, and pregabalin. Inquiry into the use of non-prescription medications revealed a daily total over-the-counter acetaminophen intake of $4875 \mathrm{mg}$. This dosing regimen had not been prescribed based on his previous medical records.

On bedside assessment, he was positioned upright in respiratory distress. His blood pressure was $98 / 61 \mathrm{~mm} \mathrm{Hg}$, pulse 119 beats per minute, temperature $35.2^{\circ} \mathrm{C}$, respiratory rate 20 breaths per minute, and oxygen saturation 97 percent while supplemented with $5 \mathrm{~L} / \mathrm{min}$ of oxygen by nasal cannula. His level of consciousness was normal with no focal neurological deficits. His abdominal examination revealed faint bowel sounds, and his abdomen was soft and diffusely tender to palpation. There was no guarding, rebound tenderness, or hepatosplenomegaly. Cardiovascular examination revealed warm and well-perfused extremities. His jugular venous pressure was at the sternal angle. He had mild pedal edema bilaterally. Heart sounds were normal, with no murmurs or extra sounds. His respiratory exam revealed increased work of breathing without wheeze. There were mild crackles over the left lung base.

Initial bloodwork is summarized in Table 1. Most notable was a profound metabolic acidosis with an elevated anion gap in addition to an acute kidney injury. His acetaminophen level was $357 \mathrm{mcg} / \mathrm{mL}$ (normal $<200 \mathrm{mcg} / \mathrm{mL}$ ). His INR was elevated at 2.17. Chest radiograph demonstrated a new hazy opacity in the left lower lobe. Given the marked abdominal pain and increased venous lactate, computed tomography angiography of the abdomen was performed, which did not show a bowel obstruction or evidence of mesenteric ischemia.

This patient presented with a HAGMA and a normal osmolar gap in the context of shock and acute kidney injury. In light of the patient's significant comorbidities, several etiologies could have caused his HAGMA. Although his initial hemodynamic parameters and hazy opacity on chest imaging were suggestive of sepsis secondary to pneumonia, his serum lactate was only mildly elevated $(2.3 \mathrm{mM})$. Chronic malnutrition raised the possibility of starvation ketosis, however, the mild increase in ketones (1.5 $\mathrm{mM}$ ) in conjunction with the lack of reported acute decline in caloric intake on history made this less likely. We believed that his acute-on-chronic renal impairment was likely insufficient to fully explain his initial anion gap of 28 . The presence of an elevated serum acetaminophen level without a history of an acute overdose thus raised the possibility of acetaminophen contributing to the HAGMA. In light of his chronic acetaminophen overuse and marked acidosis, a presumptive diagnosis of toxicity from the accumulation of 5-oxoproline was thus made and targeted management was instituted.

His initial management focused on volume resuscitation treatment of the acidosis. Isotonic fluids were rapidly administered, followed by sodium bicarbonate boluses and then a continuous infusion. $\mathrm{N}$-acetylcysteine was also administered to treat possible delayed acetaminophen toxicity. Given the possibility of 5-oxoproline toxicity, a urine organic acid assay was sent. Broad-spectrum antibiotics were administered for a possible respiratory source of sepsis.

Following discussion with the patient and family, escalation of care was ultimately declined. Nephrology consultation was obtained, and since hemodialysis was not within his goals of care, conservative measures were continued. He was admitted to the general medical ward and exhibited minimal clinical improvement. His anion gap was virtually unchanged after more than 12 hours of sodium bicarbonate infusion, and his acidemia was refractory to treatment. He developed oliguric renal failure, pulmonary edema, and cardiogenic shock with worsening mental status. Palliative care measures were instituted, and he died less than 24 hours following admission. The results of his urine organic assay returned two weeks later, the report stating that "acetaminophen metabolites [were] present," suggesting acetaminophen's role in this patient's HAGMA. Quantification of organic acids was not available. 


\section{Discussion}

Metabolic acidosis is a common, potentially serious, acid-base disturbance that requires prompt diagnosis and treatment of the underlying cause. An anion gap, defined as $\left[\mathrm{Na}^{+}\right]-\left(\left[\mathrm{Cl}^{-}\right]\right.$ $\left.+\left[\mathrm{HCO}^{3-}\right]\right)$, can be further classified into normal anion gap and high anion gap metabolic acidosis (HAGMA). Causes of a HAGMA have been classically organized into lactic acidosis, ketoacidosis (e.g., starvation, diabetic ketoacidosis), metabolites generated by toxic ingestions (e.g., ethylene glycol, methanol, salicylates), and severe chronic kidney disease. In one study of hospitalized patients with increased serum anion gaps, nearly one third were not accounted for by ketones or lactate, highlighting the need for consideration of other unidentified organic anions in the serum. ${ }^{1}$

HAGMA due to accumulation of serum 5-oxoproline (pyrogluatamic acid) is an infrequently considered phenomenon that develops in patients with a history of chronic or subacute acetaminophen use, often at therapeutic doses. ${ }^{2}$ Including our case, reports of confirmed and/or presumed diagnoses of 5-oxoprolinemia acidosis total at least 60 patients in the literature. ${ }^{3-10} \mathrm{~A}$ lack of clinician awareness and difficulty accessing a timely diagnostic assay are postulated to be the main factors contributing to its underreporting. ${ }^{6}$

5 -oxoproline is an endogenous organic acid that is an intermediate in the gamma-glutamyl cycle, an ATP-dependent six-enzyme process that transports amino acids across cell membranes. ${ }^{3}$ This cycle produces glutathione, an antioxidant amino acid that inactivates free radicals and aids in endogenous toxin excretion. ${ }^{7}$ Through negative feedback, glutathione ultimately acts on the gamma-glutamylcysteine synthetase to inhibit its production. ${ }^{11}$

Acetaminophen's toxicity is well-studied in the acute setting. The buildup of the metabolite $\mathrm{N}$-acetyl-p-benzoquinone imine (NAPQI) irreversibly binds with glutathione to form a non-toxic conjugate, resulting in depletion of hepatic glutathione reserves. ${ }^{12}$ Most often, a concomitant HAGMA is attributed to renal failure and lactate accumulation, but in cases where the anion gap remains unaccounted for, use of chronic acetaminophen has been implicated. ${ }^{13}$ With chronic use, the depletion of glutathione results in the accumulation of 5-oxoproline, an organic acid that dissociates and contributes to acidosis. ${ }^{14}$

A variety of factors may predispose patients with chronic acetaminophen ingestion to develop toxicity from 5-oxoproline. Most cases in the literature describe patients taking therapeutic doses of acetaminophen, and it is, therefore, the emergence of acute intercurrent illness that often increases a patient's susceptibility to 5-oxoprolinemia. ${ }^{8}$ Malnutrition, pregnancy, and strict vegan diets can lead to deficiency in glycine that can exacerbate the depletion of hepatic glutathione and shift the gamma-glutamyl cycle towards the generation of 5-oxoproline. ${ }^{11}$ Alcohol use disorder, liver disease, and states associated with high oxidative stress (e.g., sepsis) may also lead to deficiencies in hepatic glutathione. ${ }^{14}$ Furthermore since 5-oxoproline is renally excreted, renal insufficiency has been identified as a comorbidity which causes patients to be particularly susceptible to 5-oxoprolinemia and subsequent acidosis. ${ }^{11}$ Clinically, patients with 5-oxoprolinemia present with a constellation of symptoms, notably altered mental status, nausea, vomiting, abdominal pain, dyspnea, and malaise. ${ }^{8,11,15}$

As in this case, confirmatory testing is rarely available at the point of care, and the diagnosis is made presumptively, based on the patient's history and risk factors. This requires the clinician to be aware of the entity and the settings in which it usually occurs. Unlike this case, serum acetaminophen levels are often within the therapeutic range. ${ }^{11}$ Liver enzymes and function tests are often normal. ${ }^{16-18}$ The gold standard test, a urine organic acid profile, is often not easily available, and the result may not be available in a timely fashion. ${ }^{3}$ In our case, we ordered a urine organic acid assay and then consulted the Clinical Biochemistry service to assist in the interpretation of the result. The assay methodology was based on putative identification of the potential compounds based on a given library and of a qualitative nature, similar to previously reported cases. ${ }^{18}$ Serum testing and quantitative urine testing for 5-oxoproline was not available in our centre. These analyses are completed typically via gas chromatography. ${ }^{8}$

When all major causes of anion gap metabolic acidosis have been excluded and the clinical suspicion is high for chronic acetaminophen use, urine and plasma samples should be collected and empiric therapy initiated. Acetaminophencontaining medications should be stopped. Correction of the underlying acidosis with sodium bicarbonate and management of electrolyte derangements is also recommended. ${ }^{3}$ Due to its favourable safety profile and theoretical therapeutic role in repleting hepatic glutathione stores, administration of $\mathrm{N}$-acetylcysteine is recommended, despite the lack of evidence for its use in this setting. ${ }^{8,19}$ Hemodialysis has also been shown to accelerate the clearance of the accumulated 5-oxoproline and resolve severe acidemia. $^{20}$

Full recovery has been reported in patients who cease acetaminophen use, with normalization of the urine organic acid profile and without any apparent short- or long-term sequelae., Once clinical recovery and certain predisposing conditions (e.g., malnutrition, sepsis) are corrected, patients have been demonstrated to safely tolerate resumption of acetaminophen therapy at therapeutic doses. ${ }^{8}$ Nonetheless, as in our case, deaths have been reported from episodes of refractory acidosis associated with 5-oxoprolinuria. ${ }^{8,18}$ 


\section{Case Study}

Table 1. Laboratory Data of Patient

\begin{tabular}{|c|c|c|}
\hline Parameter & Reference Range & Patient Result \\
\hline \multicolumn{3}{|l|}{ Hematology } \\
\hline Hemoglobin (male, g/L) & $130-170$ & 107 \\
\hline White blood cell count $(\times 109 / \mathrm{L})$ & $4-10$ & 13.8 \\
\hline Platelet count (×109/L) & $130-400$ & 95 \\
\hline INR & $0.8-1.2$ & 2.17 \\
\hline PT (s) & $11-14$ & 25.9 \\
\hline \multicolumn{3}{|l|}{ Serum Chemistry } \\
\hline Sodium (mM) & $135-145$ & 138 \\
\hline Potassium (mM) & $3.5-5.0$ & 3.8 \\
\hline Chloride (mM) & 98-106 & 104 \\
\hline Bicarbonate (mM) & $24-30$ & 6 \\
\hline Creatinine (male, mM) & $70-120$ & 353 \\
\hline Anion gap (mM) & $3-11$ & 28 \\
\hline Albumin (g/L) & $35-50$ & 32 \\
\hline Glucose (mM) & $3.3-5.8$ & 3.3 \\
\hline Total calcium (mM) & $2.18-2.58$ & 2.37 \\
\hline Magnesium (mM) & $0.75-0.95$ & 1.05 \\
\hline Phosphate (mM) & $0.8-1.5$ & 2.36 \\
\hline Urea (mM) & $2.5-7.1$ & 26 \\
\hline Total bilirubin (mM) & $<26$ & 8 \\
\hline Lipase (U/L) & $<160$ & 21 \\
\hline Alkaline phosphatase (U/L) & $38-126$ & 245 \\
\hline Alanine aminotransferase (U/L) & $17-63$ & 9 \\
\hline Aspartate aminotransferase (U/L) & $18-40$ & 28 \\
\hline Lactate (mM) & $1-1.8$ & 2.3 \\
\hline Osmolality $(\mathrm{mmol} / \mathrm{kg})$ & $280-300$ & 330 \\
\hline Osmolar gap & $<10$ & 11 \\
\hline Troponin (ng/L) & $<14$ & 228 \\
\hline
\end{tabular}


Table 1. Laboratory Data of Patient (continued)

\begin{tabular}{|c|c|c|}
\hline \multirow{2}{*}{ Parameter } & \multicolumn{2}{|l|}{ Reference range } \\
\hline & $<0.6$ & 1.5 \\
\hline HbA1c (\%) & $<0.065$ & 0.062 \\
\hline \multicolumn{3}{|l|}{ Venous Blood Gas } \\
\hline $\mathrm{pH}$ & $7.32-7.42$ & 6.95 \\
\hline pCO2 (mmHg) & $41-51$ & 33 \\
\hline p02 (mmHg) & $25-40$ & 46 \\
\hline $\mathrm{HCO}$ (mM) & $22-26$ & $<8$ \\
\hline \multicolumn{3}{|l|}{ Special Tests } \\
\hline Acetaminophen level (mcg/mL) & $<200$ & 357 \\
\hline $\begin{array}{l}\text { Salicylates, ethanol, methanol,isopropanol, } \\
\text { acetone }\end{array}$ & & Not detected \\
\hline $\begin{array}{l}\text { Barbiturates, benzodiazepines, gamma- } \\
\text { hydroxybutyric acid, ibuprofen, tricyclic } \\
\text { antidepressants }\end{array}$ & & Not detected \\
\hline Broad-spectrum urine drug screen & & No substances detected \\
\hline Peripheral blood cultures $(\times 3)$ & & No growth detected. \\
\hline \multicolumn{3}{|l|}{ Urine Tests } \\
\hline Urinalysis & \multicolumn{2}{|l|}{$\begin{array}{l}\text { No ketones/glucose/nitrites. } \\
\text { Large amounts of blood, } 1.0 \text { protein, + leukocytes. }\end{array}$} \\
\hline Urine cultures & & No growth detected. \\
\hline Creatinine (uM) & & 4294 \\
\hline Albumin (mg/L) & $<24$ & 334.3 \\
\hline Urine albumin/creatinine & $<1.9$ & 78 \\
\hline Organic acid urine screen & \multicolumn{2}{|c|}{$\begin{array}{l}\text { Acetaminophen metabolites presented. Isolated elevation of adipic acid which may result from diet } \\
\text { The remainder of urine organic acids appears normal. }\end{array}$} \\
\hline
\end{tabular}

This case raises important implications for clinical practice, research, and medical education. It is important to advocate for patient awareness of acetaminophen dosing limits, but even this might not prevent the onset of 5-oxoprolemia since toxicity from acetaminophen is not always present. Over-the-counter combination products are commonly not recognized to contain acetaminophen, with nearly half of adults studied demonstrating they would inadvertently overdose in acetaminophen when using multiple acetaminophen-containing medications. ${ }^{21}$ Patient education on the adverse effects of chronic acetaminophen ingestion should be reinforced. Additionally, it is believed that many cases of toxicity from 5 -oxoprolinemia go undiagnosed. The importance of obtaining accurate medication history, including specific questioning about non-prescription drug use, is paramount. 
Table 2. Mnemonic for the Differential Diagnosis of Anion-Gap Metabolic Acidosis for The 21st Century - GOLD MARK

\begin{tabular}{|c|c|}
\hline Glycols & Ethylene and propylene \\
\hline Oxoproline & 5-oxoproline (pyroglutamic acid) \\
\hline L-lactate & Seen in hypoxia, ischemia, trauma \\
\hline D-lactate & $\begin{array}{l}\text { Occurs in the setting of short bowel syndrome often } \\
\text { post-bariatric surgery, where there is fermentation of } \\
\text { excess carbohydrates }\end{array}$ \\
\hline Methanol & And other toxins (ethanol, toluene, paraldehyde) \\
\hline Aspirin & And other salicylates \\
\hline \multicolumn{2}{|l|}{ Renal failure } \\
\hline Ketoacidosis & Seen in diabetic ketoacidosis, inadequate oral intake \\
\hline
\end{tabular}

There is a growing body of literature of case reports and case series examining 5-oxoprolinemia. Better characterization of the clinical manifestations and predisposing risk factors to toxicity from chronic acetaminophen use on a larger scale through ongoing reporting of cases of 5-oxoprolinemia will enable better evaluation of its incidence and disease course.

There is an opportunity to improve recognition of this disease through interventions with medical students and resident trainees. With the advent of enhanced laboratory abilities to detect organic acids and their precursors and the changing prevalence of HAGMA causes (e.g., rarity of iron and isoniazid poisoning), Mehta et al. proposed a contemporary mnemonic to inform the differential diagnosis of an anion gap metabolic acidosis entitled GOLD MARK (Table 2). ${ }^{22}$ Mnemonics serve as a valuable organizational framework for retention and recall of information, particularly for trainees who are in the process of developing their clinical practices. ${ }^{23}$ For those clinicians given to using mnemonics, we recommend this mnemonic to be adopted into the medical education curriculum to align trainees' diagnostic processes with today's most commonly encountered causes of HAGMA.

In conclusions, clinicians should consider 5-oxoprolemia on the differential in patients who present with an unexplained anion gap metabolic acidosis and history of subacute to chronic acetaminophen use, particularly when risk factors such as malnutrition, renal insufficiency, and sepsis are present. Treatment consists of cessation of acetaminophen-containing medications, correction of the underlying metabolic derangements, glutathione repletion with $\mathrm{N}$-acetylcysteine, and supportive care. Increased access to timely diagnostic tests for elevations in 5-oxoproline will help ensure that cases do not go unrecognized. Increased reporting will enable better evaluation of its incidence and disease course. Finally, a further systematic study of therapeutic strategies for acetaminophen-induced 5-oxoprolinemia is required.

\section{References}

1. Gabow P, Kaehney W, Fennessey P, et al. Diagnostic importance of an increased serum anion gap. N Engl J Med 1980;303(15):854-58. doi:10.1056/ NEJM198010093031505.

2. Abkur TM, Mohammed W, Ali M, et al. Acetaminophen-induced anion gap metabolic acidosis secondary to 5-oxoproline: A case report. J Med Case Rep 2014;8(1):1-4. doi:10.1186/1752-1947-8-409.

3. Hunter RW, Lawson C, Galitsiou E, et al. Pyroglutamic acidosis in association with therapeutic paracetamol use. Clin Med 2016;16(6):524-29.

4. Agrawal A, Kishlyansky M, Biso S, et al. Common, yet elusive: A case of severe anion gap acidosis. Oxford Med Case Rep 2017;2017(9):176-77. doi:10.1093/omcr/omx054.

5. Berbee JK, Lammers LA, Krediet CTP, et al. Metabolic acidosis caused by concomitant use of paracetamol (acetaminophen) and flucloxacillin? A case report and a retrospective study. Eur J Clin Pharmacol 2017;73(11):1459-65. doi:10.1007/s00228-017-2311-6.

6. Hundemer GL, Fenves AZ. Acquired 5-oxoproline acidemia successfully treated with N-acetylcysteine. Proc (Bayl Univ Med Cent) 2017;30(2):169-70.

7. Pham AQT, Xu LHR, Moe OW. Drug-induced metabolic acidosis. Adverse Drug React Bull 2017;304(1):1175-78. doi:10.1097/FAD.0000000000000025.

8. Pitt JJ, Hauser S. Transient 5-oxoprolinuria and high anion gap metabolic acidosis: Clinical and biochemical findings in eleven subjects. Clin Chem 1998;44(7):1497-503.

9. Duewall JL, Fenves AZ, Richey DS, et al. 5-Oxoproline (Pyroglutamic) acidosis associated with chronic acetaminophen Use. Baylor Univ Med Cent Proc 2010;23(1):19-20. doi:10.1080/08998280.2010.11928574.

10. Alhourani HM, Kumar A, George LK, et al. Recurrent pyroglutamic acidosis related to therapeutic acetaminophen. Am J Med Sci 2018;355(4):387-89. doi:https://doi.org/10.1016/j.amjms.2017.08.001.

11. Fenves AZ, Kirkpatrick HM, Patel VV, et al. Increased anion gap metabolic acidosis as a result of 5-oxoproline (pyroglutamic acid): a role for acetaminophen. Clin J Am Soc Nephrol 2006;1(3):441-47. doi:10.2215/ CJN.01411005.

12. Lu S. Regulation of hepatic glutathione synthesis. Semin Liver Dis 1998;18:331-43.

13. Record C, Iles R, Cohen R, et al. Acid-base and metabolic disturbances in fulminant hepatic failure. Gut 1975;16:144-49.

14. Zand L, Muriithi A, Nelsen E, et al. Severe anion gap metabolic acidosis from acetaminophen use secondary to 5-oxoproline (pyroglutamic acid) accumulation. Am J Med Sci 2012;344(6):501-504. doi:10.1097/ MAJ.0b013e318259bd45.

15. Dempsey GA, Lyall HJ, Corke CF, et al. Pyroglutamic acidemia: A cause of high anion gap metabolic acidosis. Crit Care Med 2000;28(6):1803-807. doi:10.1097/00003246-200006000-00018.

16. Reddi AS, Kunadi AR. Recurrent anion gap metabolic acidosis in a woman with vertebral disc disease. Am J Emerg Med 2011;29(8). doi:10.1016/j. ajem.2010.08.023.

17. Brooker G, Jeffery J, Nataraj T, et al. High anion gap metabolic acidosis secondary to pyroglutamic aciduria (5-oxoprolinuria): Association with prescription drugs and malnutrition. Ann Clin Biochem 2007;44(4):406-409. doi:10.1258/000456307780945769.

18. Liss DB, Paden MS, Schwarz ES, et al. What is the clinical significance of 5-oxoproline (pyroglutamic acid) in high anion gap metabolic acidosis following paracetamol (acetaminophen) exposure? Clin Toxicol 2013;51(9):817-27. doi:10.3109/15563650.2013.844822. 
19. Tailor P, Raman T, Garganta CL, et al. Recurrent high anion gap metabolic acidosis secondary to 5-oxoproline (pyroglutamic acid). Am J Kidney Dis 2005;46(1):4-10. doi:10.1053/j.ajkd.2005.03.021.

20. Luyasu S, Wamelink MMC, Galanti L, et al. Pyroglutamic acid-induced metabolic acidosis: A case report. Acta Clin Belg 2014;69(3):221-23. doi:10.1 179/2295333714Y.0000000022.

21. Wolf MS, King J, Jacobson K, et al. Risk of unintentional overdose with nonprescription acetaminophen products. J Gen Intern Med 2012;27(12):158793. doi:10.1007/s11606-012-2096-3.
22. Mehta AN, Emmett JB, Emmett M. GOLD MARK: an anion gap mnemonic for the 21st century. Lancet 2008;372(9642):892. doi:10.1016/ S0140-6736(08)61398-7.

23. Koeckeritz JL, Hopkins KV, Merrill AS. Memory and how it works. Nurse Educ 2004;29(2):75-79.

\section{ESIM

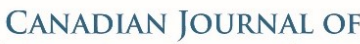 \\ General Internal Medicine \\ LA REVUE CANADIENNE DE MÉDECINE INTERNE GÉNÉRALE}

\section{Thank You To All Our 2018/2019 Article Reviewers}

Dr. Amna Ahmed

Dr. Hector Baillie

Dr. Elvira Bangert

Dr. Kerstin deWitt

Dr. Rodgrio Cavalcanti

Dr. James Douketis

Dr. Donald Echenberg

Dr. Jim Gibson

Dr. Will Harper

Dr. Rick Ikesaka

Dr. Christopher Hillis

Dr. Murray Krahn

Dr. Wilson Kwong

Dr. Mitchell Levine

Dr. Tom Macmillan
Dr. Tushar Suryakant M alavade

Dr. Lindsay M elvin

Dr. John Neary

Dr. Kara Nerenberg

Dr. Tim O'Shea

Dr. Mohamed Panju

Dr. Casey Park

Dr. Ameen Patel

Dr. Winnie Sia

Dr. Rupal Shah

Dr. Linda Snell

Dr. Michel Sauve

Dr. John You

Dr. Ben Wilson

Dr. Robert Wu 